\title{
Emergent Chimney Stent to Treat Left Main Occlusion Following Valve-In-Valve Transfemoral Aortic Implantation Chimney Stent Following Valve-In-Valve TAVI
}

\author{
Luis Fernandez Gonzalez*, Roberto Blanco Mata, Koldobika Garcia San Roman and Juan \\ Alcibar Villa \\ Interventional Cardiology,Cruces University Hospital, Baracaldo, Spain
}

Received: 04 December, 2017; Accepted: 08 January, 2018; Published: 15 January, 2018

*Corresponding author: Luis Fernandez Gonzalez, Interventional Cardiology, Cruces University Hospital, Baracaldo, Spain; E-mail id: Iuisfg82@ hotmail.com

\begin{abstract}
Implantation of transcatheter valves inside degenerated surgical bioprosthesis is nowadays a very good alternative to redo a new cardiac surgery especially in high risk patients. Although procedural success is achieved in most of the patients, these procedures are associated with few potential risks such as coronary occlusion. It is very important to perform an exhaustive study prior to the valve implantation so as to prevent possible fatal complications. In this paper we show that in case of coronary occlusion one possibility to solve it is emergent percutaneous coronary intervention.
\end{abstract}

Keyword: Aortic Valve Disease, Percutaneous Intervention, Balloon Angioplasty, Complications, Structural Heart Disease Intervention

\section{Introduction}

Most of the surgical valves being implanted during the past decade are bioprosthetic with limited durability. Implantation of transcatheter valves (Valve in Valve TAVI) inside de-generated surgical bioprosthesis is reported as an alternative to repeat surgery [1]. Although procedural success is achieved in most of the patients, this procedures are associated with few potential risks such as coronary occlusion [2-4]. Coronary obstruction is associated with high rate of mortality. In this paper we describe a case of left coronary obstruction following Valve in Valve (VIV) TAVI procedure solved by Percutaneous Coronary Intervention (PCI) of left main.

\section{Case Report}

A 82 years old woman with previous aortic valve replacement with a bioprosthesis Carpentier Perimount 19 and Kallangos 28 mitral ring 10 years ago, was referred to our unit to undergo to a valve in valve TAVI for symptomatic bioprosthesis degeneration. She was documented to be a high risk candidate for future repeat surgery due to comorbilities (Hypertension, cognitive impairment, atrial fibrillation, poor mobility).

Transthoracic echocardiography showed severe stenosis (mean trans-aortic gradient $41 \mathrm{~mm} \mathrm{Hg}$, aortic valve orifice 0,6 $\mathrm{cm}^{2}$ ) with mild aortic regurgitation and preserved left ventricular function with no significant mitral insufficiency through the mitral ring. Coronary angiography documented no coronary disease but a close proximity between left coronary artery and bioprosthetic valve. Cardiac Tomography (CT) showed high risk of coronary occlusion (8 $\mathrm{mm}$ height between aortic ring and left coronary ostium) and in order to that issue we protected the left coronary artery with a guide wire during the valve implantation. (Figure: Panel A)

An Edwards XT 20 (Edwards Life sciences, Irvine CA) mm was successfully deployed under rapid pacing after systematically checking blood flow in the left coronary artery through-out the procedure, being the left main patent at the end of the procedure, because of that we decided to remove the rescue guide wire (Figure: Panel B)

Ten minutes later, when we was closing the femoral artery with the Proglyde (Abbott) sys-tem, a cardiac arrest occurred, we showed anterior ST elevation in ECG with deterioration un LV function in the echo and suspected dynamic occlusion of left main that was con-firmed with non selective coronary angiography with a Judkins left 4 catheter (Figure: Panel C, D, E)

We attempted emergency PCI to left main by using 2 guide wires (LAD and Circunflex), first of all we predilate left coronary ostium with a non compliant balloon of $3.5 \mathrm{~mm}$ of diameter to restore coronary flow that underwent improving of hemodynamic situation of the patient (Figure: Panel F). After removing the balloon we continued showing dynamic coronary occlusion due to an excess of tissue of the valve and therefore we needed to deploy a drug eluting zotarolimus stent ( $4 \mathrm{x} 18$ ) so as to get enough radial force to overwhelm valve compression and secure left main flow by using a technique called Chimney stent which is used during EVAR procedures for renal artery salvation (Figure: Panel G,H). Patient's hemodynamics rapidly improved after stent implantation and could be discharged after 5 days without complications. 6 months later no angina symptoms and normal LV and TAVI function. 


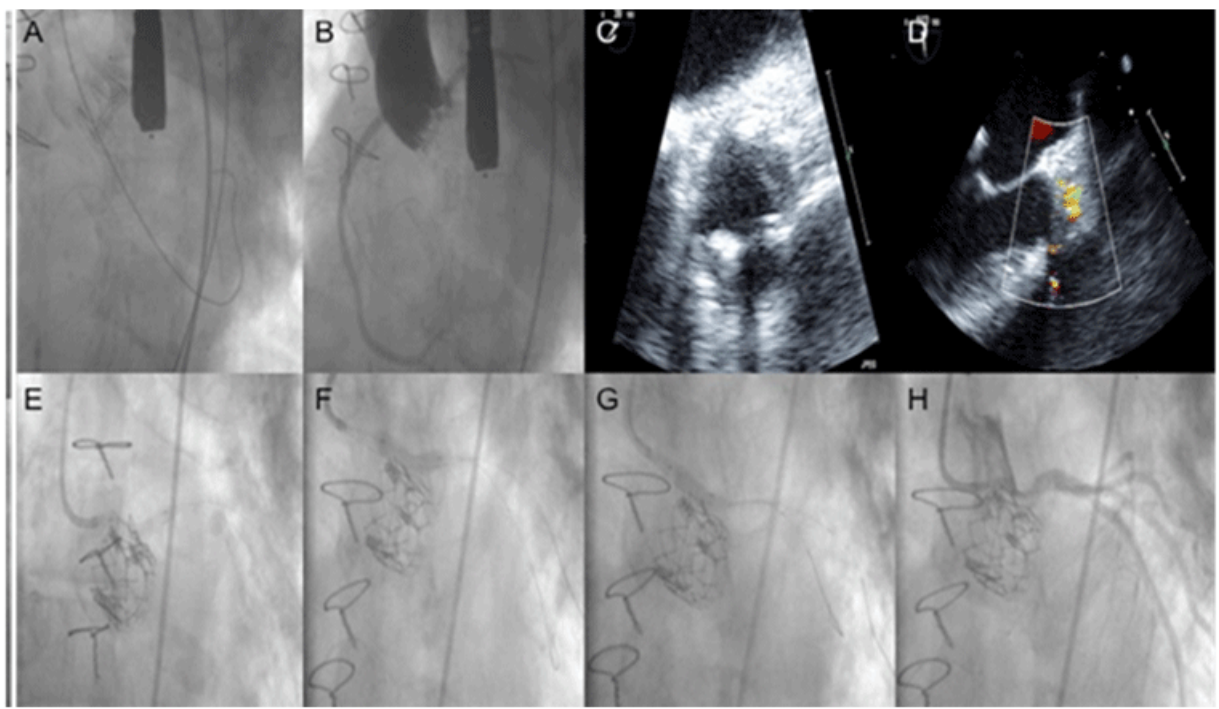

Figure : A. Coronary guide wire placed in LAD before valve implantation. B. Angiography that shows left coronary flow compromise minutes after VIV implantation. C and D. Echo image that shows left coronary ostium dynamic occlusion. E. Selective angiography with a guide catheter. F. Predilation of left coronary ostium with a non compliant ballooon. G. Zotarolimus stent implantation with postdilation to achieve the "Chimney form". H. Final result with normal coronary flow.

\section{Discussion}

Acute coronary occlusion of a coronary artery is an infrecuent but life threatening complication following TAVI mainly due to the displacement of a calcific leaflet towards the coronary ostia. Initially it had been suggested that the presence of an in situ surgical bioprosthesis could avoid this complication, but acute coronary obstruction has been describe in several cases of TAVI valve in valve $[1,2]$. The knowledge of the intrinsic properties of the surgical bioprosthesis and CT before the procedure to determine the distance between the coronary ostia and prosthesis is essential in those cases. There are other possible anatomic risk factors for coronary obstruction that have been reported such as narrow sinotubular junction, narrow sinuses of Valsalva or previous root repair with coronary reimplantation [2]. Regarding the bioprothesis factors, the supra-annular position, the high leaflet profile, the internal stent frame (Mitroflow, Trifecta...) or bulky leaflet could deter-mine the possible coronary occlusion during the procedure. We have to take for granted all of these factors prior the VIV procedure so as to avoid a possible coronary obstruction [3]. In the majority of the cases of coronary occlusion after VIV procedure published in the past, the possible solution was immediate cardiac surgery in order to perform a aorto-coronary bypass but the case that we are referring in this paper could be solved with a PCI using the "Chimney" technique originally describe for renal and mesenteric preservation after EVAR with an excellent outcome for the patient [5]. Although applied in a different clinical and procedural setting, the same principles used in renal and mesenteric salvage could be used to treat a serious life-threatening complication for VIV interventions. In cases of high risk of coronary occlusion during de VIV procedure it could even be possible to perform PCI previous to the procedure but we have no experience with this type of practice.

\section{Conclusion}

Transcatheter aortic VIV in stentless surgical bioprosthetic valves is associated with an increased risk of left main compromise. Detailed pre-procedural planning and understanding of the surgical bioprosthetic valve and its relation to the surrounding anatomic structures is very important, especially with the coronary ostia. In case of coronary occlusion a less invasive option to solve it could be PCI with a stent to get enough radial force to maintain coronary flow.

\section{References}

1. Gurvitch R, Cheung A, Bedogni F, Webb JG. Coronary Obstruction following transcatheter aortic valve-in-valve implantation for failed surgical Bioprostheses. Catheter Cardiovasc Interv. 2011;77(3):43944. doi: $10.1002 / \mathrm{ccd} .22861$

2. Dvir D, Leipsic J, Blanke P, Ribeiro HB, Kornowski R, Pichard A, et al. Coronary obstruction in transcatheter aortic valve-in-valve implantation: preprocedural evaluation, device selection, protection, and treatment.. Circ Cardiovasc Interv. 2015;8(1). pii: e002079. doi: 10.1161/CIRCINTERVENTIONS.114.002079

3. Urena M, Nombela-Franco L, Doyle D, De Larochelliere R, Dumont E, Villeneuve J, et al. Transcatheter aortic valve implantation for the treatment of surgical valve dys-function ("valve-in-valve"): assesing the risk of coronary obstruction. J Card Surg. 2012;27(6):682-685. doi: 10.1111/jocs.12003

4. Chakravarty T, Jilaihawi H, Nakamura M, Kashif M, Kar S, Cheng W, et al. Pre-emptive positioning of a coronary stent in the left anterior descending artery for left main protection: A prerequisite for transcatheter aortic valve-in-valve implantation for failing stentless bioprosthesis? Catheter Cardiovasc Interv. 2013;82(4):E630-636. doi: 10.1002/ccd.25037

5. Liu B, Pan H, Song X, Liu C, Wu W, Chen Y, et al. Chimney stents for endovascular repair of juxtarenal aortic aneurysms with unfa-vourable anatomy. Int Angiol. 2013;32(3):307-311. 